\title{
Relationship between body habitus and image quality and radiation dose in chest x-ray examinations: A phantom study
}

Purpose: To evaluate the influence of being overweight on image quality (IQ), radiation dose and acquisition parameters when undertaking adult chest $\mathrm{x}$-ray (CXR) examinations using routine acquisition protocols.

Methods: The Lungman chest phantom, with and without chest plates, was used to simulate the chest region for larger size and average adult patients, respectively. Radiographic acquisitions were conducted using $17 \mathrm{x}$-ray machines located in eight hospitals using their routine clinical protocols. IQ was assessed using relative visual grading analysis (VGA) and 2 alternative forced choice (2AFC) by six observers. Incident air kerma (IAK) was measured using a solid-state dosimeter.

Results: IQ mean (range) scores between the hospitals were 16.2 (12.0 to 21.3) with a 56.0\% difference and 20.9 (14.1 to 23.6) with a $50.2 \%$ difference for the standard and larger size phantoms, respectively. IAK mean (range) scores $63 \mu \mathrm{Gy}$ (19 to $136 \mu \mathrm{Gy}$ ) with a $150 \%$ difference and $159 \mu \mathrm{Gy}$ ( 27 to $384 \mu \mathrm{Gy}$ ) with a $173 \%$ difference for the standard and larger size phantoms, respectively. The chest plates had a significant negative impact on IQ ( $\mathrm{P}=0.001)$ and lead to an increased in IAK by approximately $50 \%$.

Conclusion: Visual measures of IQ and IAK showed large differences between hospitals for standard and larger phantom sizes; differences within the hospitals was lower. Overall, Lungman with chest plates was found to degrade IQ and increase radiation dose by a factor of two. Further optimisation is required especially for the larger sized patient's imaging protocols for all eight hospitals.

\section{Keywords:}

Image quality, radiation dose, dose optimisation, adult chest radiography, obesity, overweight. 


\section{Introduction}

The number of overweight and obese people have increased rapidly over recent years in European Union (EU) countries. In 2014, it was reported that around 51.6\% of the EU's residents were overweight [1]. Obesity is classified as a global epidemic according to the World Health Organization (WHO) [2] and it increases the hazard of developing diseases which can result in more medical procedures, including x-ray imaging. In comparison to standard weight people, obese people have larger radiation doses for the same examinations [3].

In radiography, practical difficulties often arise with imaging overweight and obese patients and there is an associated increase in radiation dose and image quality (IQ) reduction [4]. Problems can include additional x-ray beam attenuation, increased scatter radiation and longer exposure times which can result in motion artefacts [4-6]. X-ray images from patients with large body habitus are often of lower IQ and practitioners need research evidence regarding adjustments to both pre-acquisition and post-acquisition imaging parameters [7].

The Commission of the European Communities (CEC) [8] has published guidelines on adult radiographic imaging which includes the chest $\mathrm{x}$-ray (CXR) examination. These guidelines provide information on the selection of optimal acquisition parameters, but it was designed only for standard sized patients and currently there are a lack of formal guidelines or recommendations available for undertaking CXRs on overweight or obese patients, even though research on overweight obese patients has since occurred (e.g. Moore et al [9]). A further limitation of the CEC guidelines is that they were developed in an era of analogue film/screen systems.

Various studies [10-12] have evaluated IQ and radiation dose between hospitals. However, they have limitations. For example, they were carried out using physical phantoms with uniform backgrounds; consequently they have been criticised in the literature for not considering the effect of the anatomical noise arising from overlying human anatomy [13]. Whilst not a limitation in itself, the studies were carried out for only averaged size patients and larger size patients were not considered. Consequently, two questions remain unanswered in the literature: for CXR, 1) do routine imaging protocols for larger size patients differ from those of average size patients? 2) do IQ and radiation dose values differ between average and larger size patients when using routine imaging protocols?

The purpose of this study was to evaluate the influence of phantom size, represented by additional fat thickness, on CXR IQ and radiation dose in eight United Kingdom (UK) hospitals. Evaluations 
utilised an anthropomorphic phantom, routine clinical protocols and digital radiography (DR) and computed radiography (CR) imaging systems. To our knowledge, this is the first study that has sought to investigate the influence of patient size on IQ and radiation when using routine imaging protocols for adult CXR examinations.

\section{Method}

This prospective phantom study was conducted using seventeen diagnostic x-ray machines situated in eight UK hospitals. Quality control tests, based on the Institute of Physics and Engineering in Medicine (IPEM) report 91 [14] were conducted on the x-ray machines and they included an assessment of radiation dose output variation with tube voltage $(\mathrm{kV})$, tube current $(\mathrm{mA})$, and time, and the overall reproducibility. The results indicated that the machines were fit for routine clinical use with data falling within expected tolerances.

\subsection{Phantom}

The Lungman adult chest phantom (Kyoto Kagaku, Japan) is a multipurpose anthropomorphic chest phantom (Fig. 1). It has a dimensional structure that permits the simulation of posteroanterior (PA) and lateral chest features. It provides an anatomical model of a torso with soft tissue substitute materials and artificial bones of absorption ratios extremely close to human tissue. It has a removable mediastinum and a pulmonary vessel structure [15]. It has a chest girth of $94.0 \mathrm{~cm}$, with dimensions of $43.0 \mathrm{~cm}(\mathrm{w})$ x $40.0 \mathrm{~cm}$ (d) x $48.0 \mathrm{~cm}$ (h), its weight is approximately $18.0 \mathrm{Kg}$ [16]. Anterior and posterior chest plates (each $6 \mathrm{~cm}$ thick, representing human adipose tissue) can be added to the Lungman phantom to simulate a larger body size (Fig. 1a) [16]. 

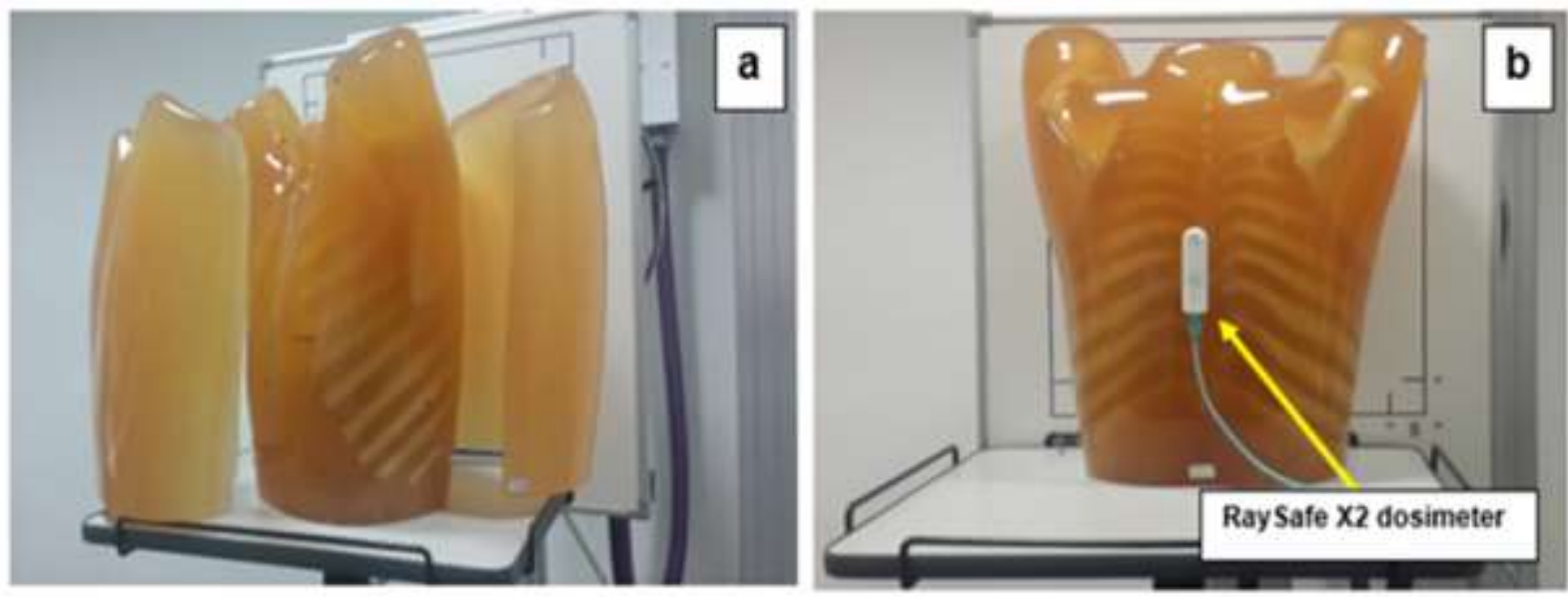

Fig. 1. The multipurpose anthropomorphic chest phantom "Lungman" (Kyoto Kagaku Co., Japan). (a) The phantom with the chest plates to simulate a larger size patient. (b) The experimental set up of the Lungman phantom experiment, positioned for PA chest radiographic examination.

\subsection{Image acquisition}

The Lungman phantom, with and without chest plates, was used to simulate the chest region for average and larger size adult patients. First, the phantom without the chest plates was utilised to acquire CXR images using the $\mathrm{x}$-ray machines and their local routine imaging protocols (Table 1) . Local protocols were defined as the default acquisition parameters routinely used on each x-ray unit. The phantom was positioned at the centre of primary radiation field and the x-ray beam was collimated to include all essential anatomical detail; relative position of phantom and collimated field remained constant throughout the experiment. Further image acquisitions, using Lungman together with the chest plates, were undertaken to simulate a larger size patient. Image acquisitions were again obtained using local protocols (Table 1). Phantom images were coded and collected from the $\mathrm{x}$-ray machines in DICOM format. 
Table 1. A summary of x-ray machine types and routine imaging protocols used for standard and larger size phantom.

\begin{tabular}{|c|c|c|c|c|c|c|c|c|c|c|c|c|}
\hline $\mathrm{H}^{1}$ & $X^{2}$ & $\begin{array}{c}X \\
\text { type }\end{array}$ & $\begin{array}{c}\mathrm{X} \\
\text { manufacturer }\end{array}$ & $\begin{array}{c}\mathrm{D}^{3} \\
\text { type }\end{array}$ & $\begin{array}{c}\mathrm{D} \\
\text { manufacturer }\end{array}$ & $\begin{array}{l}\text { Exposure } \\
\text { control } \\
\left(\mathrm{S}^{7} / \mathrm{L}^{8}\right)\end{array}$ & $\begin{array}{l}\text { Grid } \\
(\mathrm{S} / \mathrm{L})\end{array}$ & $\begin{array}{l}\text { Additional } \\
\text { filtration } \\
(\mathrm{mm})(\mathrm{S}=\mathrm{L})\end{array}$ & $\begin{array}{l}\mathrm{kVp}^{10} \\
(\mathrm{~S} / \mathrm{L})\end{array}$ & $\begin{array}{c}\mathrm{KVp} \\
\text { increase } \\
(\%)\end{array}$ & $\begin{array}{c}\mathrm{mAs}^{11} \\
(\mathrm{~S} / \mathrm{L})\end{array}$ & $\begin{array}{c}\mathrm{mAs} \\
\text { Increase } \\
(\%)\end{array}$ \\
\hline 1 & 1 & Static & Philips & $\mathrm{IDR}^{4}$ & Philips & $\mathrm{AEC} \mathrm{AEC}^{9}$ & Yes/Yes & $\begin{array}{c}0.1 \mathrm{Cu}+1.0 \\
\mathrm{Al}\end{array}$ & $125 / 125$ & 0.0 & $0.9 / 2.6$ & 172.1 \\
\hline \multirow[t]{4}{*}{2} & 1 & Mobile & Carestream & IDR & Carestream & Manual/Manual & Yes/Yes & No & $110 / 120$ & 9.1 & $2.8 / 4.0$ & 42.8 \\
\hline & 2 & Static & Siemens & IDR & Siemens & AEC/AEC & Yes/Yes & $0.1 \mathrm{Cu}$ & $125 / 125$ & 0.0 & $1.2 / 2.4$ & 100.0 \\
\hline & 3 & Static & Carestream & $\mathrm{DDR}^{5}$ & Carestream & AEC/AEC & Yes/Yes & No & $125 / 125$ & 0.0 & $1.4 / 3.8$ & 166.6 \\
\hline & 4 & Static & Samsung & IDR & Samsung & AEC/AEC & Yes/Yes & No & $125 / 130$ & 4.0 & $2.4 / 5.2$ & 116.6 \\
\hline \multirow[t]{5}{*}{3} & 1 & Static & Siemens & IDR & Siemens & Manual/Manual & $\mathrm{No} / \mathrm{No}$ & $0.2 \mathrm{Cu}$ & $96 / 96$ & 0.0 & $1.6 / 2.0$ & 25.0 \\
\hline & 2 & Static & Siemens & IDR & Siemens & Manual/Manual & $\mathrm{No} / \mathrm{No}$ & $0.2 \mathrm{Cu}$ & $96 / 96$ & 0.0 & $1.6 / 2.0$ & 25.0 \\
\hline & 3 & Static & Siemens & IDR & Siemens & Manual/Manual & $\mathrm{No} / \mathrm{No}$ & $0.2 \mathrm{Cu}$ & $96 / 96$ & 0.0 & $1.6 / 2.2$ & 37.5 \\
\hline & 4 & Static & Siemens & IDR & Siemens & Manual/Manual & $\mathrm{No} / \mathrm{No}$ & $0.2 \mathrm{Cu}$ & $96 / 96$ & 0.0 & $1.6 / 2.0$ & 25.0 \\
\hline & 5 & Static & Siemens & IDR & Siemens & Manual/Manual & $\mathrm{No} / \mathrm{No}$ & $0.2 \mathrm{Cu}$ & $96 / 96$ & 0.0 & $1.6 / 2.0$ & 25.0 \\
\hline \multirow[t]{2}{*}{4} & 1 & Static & Philips & DDR & Philips & AEC/AEC & Yes/Yes & $\begin{array}{c}1.0 \mathrm{Al}+0.1 \\
\mathrm{Cu}\end{array}$ & $125 / 125$ & 0.0 & $1.4 / 4.1$ & 195.0 \\
\hline & 2 & Static & Philips & $\mathrm{CR}^{6}$ & Carestream & AEC/AEC & Yes/Yes & No & $125 / 125$ & 0.0 & $2.8 / 7.3$ & 161.4 \\
\hline 5 & 1 & Static & Siemens & $\mathrm{CR}$ & Carestream & Manual/AEC & $\mathrm{No} / \mathrm{Yes}$ & No & $85 / 96$ & 12.9 & $2.8 / 6.0$ & 117.1 \\
\hline \multirow[t]{2}{*}{6} & 1 & Static & Philips & DDR & Philips & AEC/AEC & Yes/Yes & No & $125 / 125$ & 0.0 & $1.4 / 4.1$ & 192.8 \\
\hline & 2 & Static & Philips & DDR & Philips & AEC/AEC & Yes/Yes & No & $125 / 125$ & 0.0 & $1.1 / 3.3$ & 200.0 \\
\hline 7 & 1 & Static & Carestream & DDR & Carestream & AEC/AEC & Yes/Yes & No & $125 / 125$ & 0.0 & $0.8 / 2.5$ & 212.5 \\
\hline 8 & 1 & Static & Siemens & $\mathrm{CR}$ & Carestream & Manual/Manual & $\mathrm{No} / \mathrm{No}$ & No & $113 / 121$ & 7.0 & $2.0 / 3.2$ & 60.0 \\
\hline
\end{tabular}

${ }^{1} \mathrm{H}$ : Hospital; ${ }^{2} \mathrm{X}$ : x-ray machine; ${ }^{3} \mathrm{D}$ : detector; ${ }^{4} \mathrm{IDR}$ : indirect digital radiography; ${ }^{5} \mathrm{DDR}$ : direct digital radiography; ${ }^{6} \mathrm{CR}:$ computed radiography $;{ }^{7} \mathrm{~S}$ : standard size phantom; ${ }^{8} \mathrm{~L}$ : larger size phantom; ${ }^{9} \mathrm{AEC}$ : automated exposure control; ${ }^{10} \mathrm{kVp}$ : kilovoltage peak; ${ }^{11} \mathrm{mAs}$ : milliampere second. 


\subsection{Image quality evaluation}

Ethical approval for the visual IQ evaluations was granted from the University of Salford (HSR1617-76). IQ for 17 images from each of the standard and larger size phantom previously acquired from the different $\mathrm{x}$-ray machines (34 images in total) were assessed visually by six qualified diagnostic radiographers with clinical experience ranging from 5 to 18 years. This included relative visual grading analysis (VGA) and, separately, 2 alternative forced choice (2AFC).

For relative VGA a 3 point Likert scale (worse, equal, better) was used along with bespoke computer software to display images and capture observer IQ responses [17]. IQ scoring criteria were based on the CEC [8] and a further published study [18]; these are presented in Table 2. These criteria focused on how well an image demonstrates anatomical structures. However, pathology identification or the decision whether the image quality is adequate for diagnosis were not considered.

For relative VGA, images were presented to observers on two computer monitors, on the left monitor there was a fixed reference image and on the right monitor the experimental images were displayed in a random order. Monitor characteristics are as follows, 5 mega-pixel DOME E5 (NDSsi, Santa Rosa, CA; 2048 by 2560 pixels) calibrated to the DICOM Grey Scale Display Function (GSDF) Standard [17]. The reference image was chosen by consensus opinion of two experienced clinicians who interpret images as part of their normal clinical routine. The reference image had 'average' quality in comparison with the other images; this was to ensure that all of the three points on the Likert scale were utilised in the relative VGA assessments [18,19]. Observers were not permitted to manipulate windowing and magnification of the image. This was to ensure that any changes in IQ were determined by the quality of the image itself and not because of image manipulation / post-processing. The ambient room lighting was $<8$ lux. The overall relative VGA IQ score for each image was calculated by adding the scores from all of the six IQ criteria (Table 2). The final IQ score for each image was determined by taking the average IQ score from the six observers [20]. Along with average values, standard deviation (SD) values were also determined.

For investigating the influence of phantom thickness (represented by the chest plates) on IQ, a 2AFC method was used. This involved viewing two images from the Lungman phantom, one with and one without chest plates, both acquired from the same x-ray machine and presented in an 
anonymised fashion on the two monitors. Both images, with and without chest plates, had been acquired using the routine imaging protocols for each machine. For the paired images, observers were asked to rate each image on a binary scale, as follows: 1, which image which has the better IQ (compared with the other image); 0, for the image that has lower IQ. The observers were asked to consider the criteria in Table 2 when reaching their decision.

Table 2. Six image criteria that were utilised for IQ assessments using a relative VGA method [8,18].

\begin{tabular}{|c|l|}
\hline Item & \multicolumn{1}{|c|}{ Criteria } \\
\hline 1 & $\begin{array}{l}\text { Demonstration of the vascular pattern in the whole lung, particularly } \\
\text { the peripheral vessels. }\end{array}$ \\
\hline 12 & Visually sharp demonstration of the trachea and proximal bronchi. \\
\hline 3 & Visually sharp demonstration of the borders of the heart and aorta. \\
\hline 4 & $\begin{array}{l}\text { Visually sharp demonstration of the diaphragm and lateral } \\
\text { costo-phrenic angles. }\end{array}$ \\
\hline 5 & Visualisation of the retrocardiac lung and the mediastinum. \\
\hline 6 & Visualisation of the spine through the heart shadow. \\
\hline
\end{tabular}

\subsection{Dosimetry}

A solid-state dosimeter (RaySafe X2, Unfors Ray Safe AB, Billdal, Sweden) was used to measure the incident air kerma (IAK) at the surface of the phantom at the point of entry of the x-ray beam central ray (Fig. 1b). The dosimeter has a reported accuracy of $\pm 5 \%$ compared to the calibrated values and QC results fell within manufacturer tolerances. Exposure was repeated three times and the average value calculated to reduce random error. This approach was only utilised for manual exposure control; for exposures using automated exposure control (AEC) a simple extrapolation technique was used to estimate IAK. The latter was based on the estimation the IAK values from the post-exposure milliampere second (mAs) recorded at the point of acquisition. IAK values which corresponded to their respective mAs values were recorded using the solid-state dosimeter. From the graphical representation of IAK versus mAs, a best fit line and resultant regression equation (equation (1)) were generated to provide a method for estimating the IAK from post- 
exposure mAs values obtained from the phantom but without the presence of the solid-state dosimeter.

$$
Y=B X
$$

Where: $\mathrm{X}$ : represents the applied value of $\mathrm{mAs}$; Y: represents the resulting value of IAK; B represents the gradient and means that for every increase of $1 \mathrm{mAs}(\mathrm{X})$, the IAK value (Y) will increase by the value of B. B values are different for each X-ray machine used and are illustrated in Table 3.

Table 3. The gradient (B) values used in equation (1) for calculating IAK from the mAs values across the X-ray machines that used AEC.

\begin{tabular}{|c|c|}
\hline X-ray machine code & Slope (B) value \\
\hline H1X1 & 86.3 \\
\hline H2X2 & 85.3 \\
\hline H2X3 & 126.0 \\
\hline H2X4 & 129.8 \\
\hline H4X1 & 107.9 \\
\hline H4X2 & 119.8 \\
\hline H5X1 & 125.0 \\
\hline H6X1 & 148.6 \\
\hline H6X2 & 143.9 \\
\hline H7X1 & 126.6 \\
\hline
\end{tabular}




\subsection{Data analysis}

SPSS software version 22.0 (IBM Inc, Armonk, New York, US) was utilised for data analysis. The Shapiro-Wilk test was used to examine data normality. Tests showed that IAK and IQ for both the standard and larger size phantoms were normally distributed. Consequently, Pearson's statistic was utilised for investigating the degree of correlation between IQ and the corresponding IAK for both phantom sizes separately. Unpaired t-test was used to test the hypotheses that phantom thickness has no statistically significant effect on IAK. Results of Shapiro-Wilk test indicated that the IQ scores resulting from rating a binary scale 0 or 1 by observers is not normal distributed. A Mann-Whitney test was therefore utilised to test the hypotheses that phantom thickness has no statistically significant difference on IQ. The interpretation of the strength of the correlation (r) was conducted based on literature [21,22], in which $r=0.10$ to 0.29 (small), $r=0.30$ to 0.49 (medium), and $\mathrm{r}=0.50$ to 1.00 (large). To investigate the inter-observer variability for the IQ evaluations, an inter-class correlation coefficient (ICC) was utilised. ICC values less than 0.50 indicate poor reliability, values from 0.50 to 0.75 indicate moderate reliability, values from 0.75 to 0.90 indicate good reliability and finally values greater than 0.90 indicates excellent reliability $[23,24]$.

\section{Results}

Relative VGA IQ data are presented as a series of bar charts (Fig. 2. and 3). Hospitals and x-ray machines are coded using two letters and one number: the letter $(\mathrm{H})$ refers to the hospital and the letter $(\mathrm{X})$ refers to the $\mathrm{X}$-ray machine i.e. Hospital 1, X-ray machine 1 would be H1X1. IAK data are presented on the graphs as a dashed line against the corresponding IQ values. The error bars for IQ in Fig. 2. and 3 represent the SDs obtained from visual evaluation by six observers. Table 1 describes the examination techniques and acquisition parameters used for the averaged and larger size patients, both between and within the hospitals. Fig. 2 and 3 compares the scores for the IQ against the corresponding IAK values, between and within hospitals, acquired using the standard and larger size phantoms, respectively. Regarding the standard size phantom, IQ scores between the hospitals ranged from 12.0 (H8X1) to $21.3(\mathrm{H} 2 \mathrm{X} 3$ ) (mean 16.3) with a $56.0 \%$ difference. Within hospital comparisons, the range was lower, from 15.0 (H2X1) to 21.3 (H2X3) (mean 18.1) with only a $34.8 \%$ difference. The $3^{\text {rd }}$ quartile IQ between the hospitals was 17.0. IAK values 


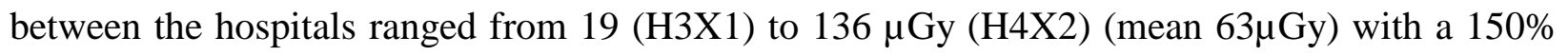
difference. The IAK range within the hospitals was smaller, from 61 (H4X1) to $136 \mu \mathrm{Gy}$ (H4X2) (mean $98 \mu \mathrm{Gy}$ ) with a $76 \%$ difference. The third quartile IAK value between the hospitals was $89 \mu \mathrm{Gy}$.

Pearson's correlation demonstrated in a moderate positive, non-significant, correlation $\mathrm{r}=0.34$ $(\mathrm{p}=0.17)$ between IAK and IQ. Good inter-observer IQ agreement was observed (ICC $=0.97$; 95\%CI: 0.61 to 0.91$)$.

In terms of the larger size phantom, relative VGA IQ scores between the hospitals ranged from 14.2 (H8X1) to 23.7 (H2X3) (mean 20.9) with a 50.2\% difference. Within the hospitals, the range was lower from $16.8(\mathrm{H} 3 \mathrm{X} 2)$ to $21.5(\mathrm{H} 3 \mathrm{X} 1)$ (mean 18.6) with a smaller $24.4 \%$ difference. The $3^{\text {rd }}$ quartile IQ scores between the hospitals was 23.0. IAK values between the hospitals ranged from 27 (H3X1) to $384 \mu \mathrm{Gy}$ (H4X2) (mean $159 \mu \mathrm{Gy}$ ) with a $173 \%$ difference. The range of IAK

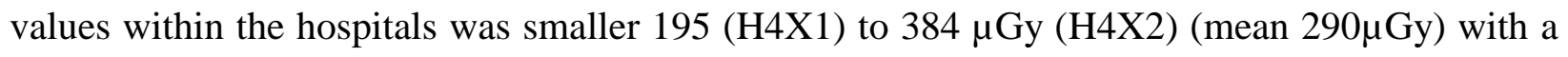
$65 \%$ difference. The $3^{\text {rd }}$ quartile IAK values between the hospitals was $213 \mu \mathrm{Gy}$. Pearson's correlation demonstrated a larger statistically significant positive correlation between IAK and IQ $(\mathrm{r}=0.60 ; \mathrm{p}=0.01)$. Good inter-observer agreement was also reported for the IQ evaluations, (ICC $=0.87$; 95\%CI: 0.74 to 0.94). Fig. 4 illustrates the influence of phantom thickness on 2AFC IQ, both between and within hospitals. The Mann-Whitney test results showed that there was a statistically significant difference $(\mathrm{P}=0.001)$ in IQ between the larger size and standard size phantom images. Fig. 5 illustrates the influence of phantom thickness on IAK, again both between and within hospitals. Results from the unpaired t-test demonstrated that there was a significant difference in IAK between the larger and standard size phantom acquisitions ( $\mathrm{P}=0.002)$. 


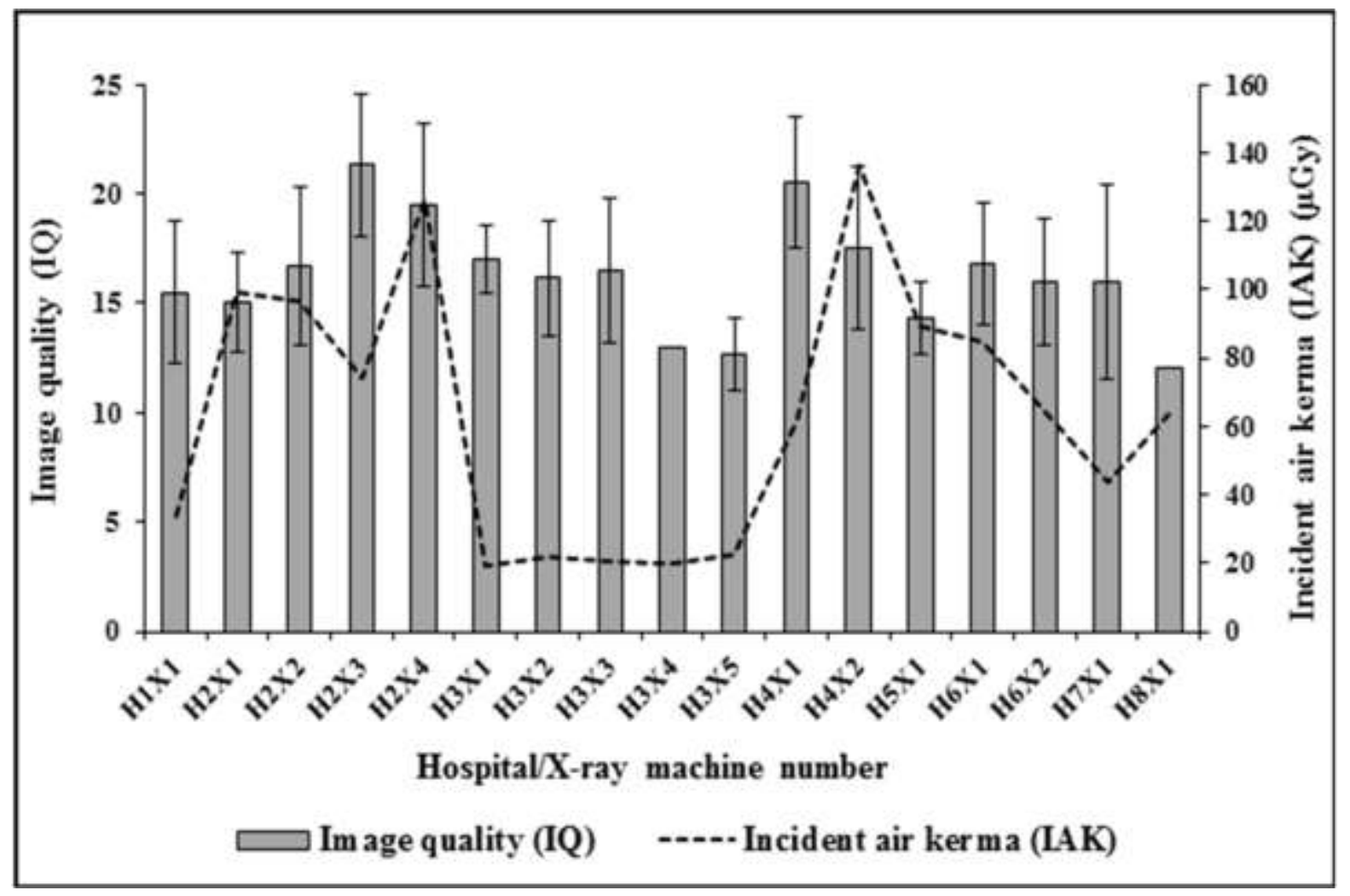

Fig. 2. A comparison of IQ values against IAK for the standard size phantom images. The error bars in this chart represent the SD in IQ obtained from the visual evaluations. The dashed line represents the average IAK value that resulted from measuring three repeated radiation exposures. $\mathrm{H}=$ hospital; $\mathrm{X}=\mathrm{x}$-ray machine. 


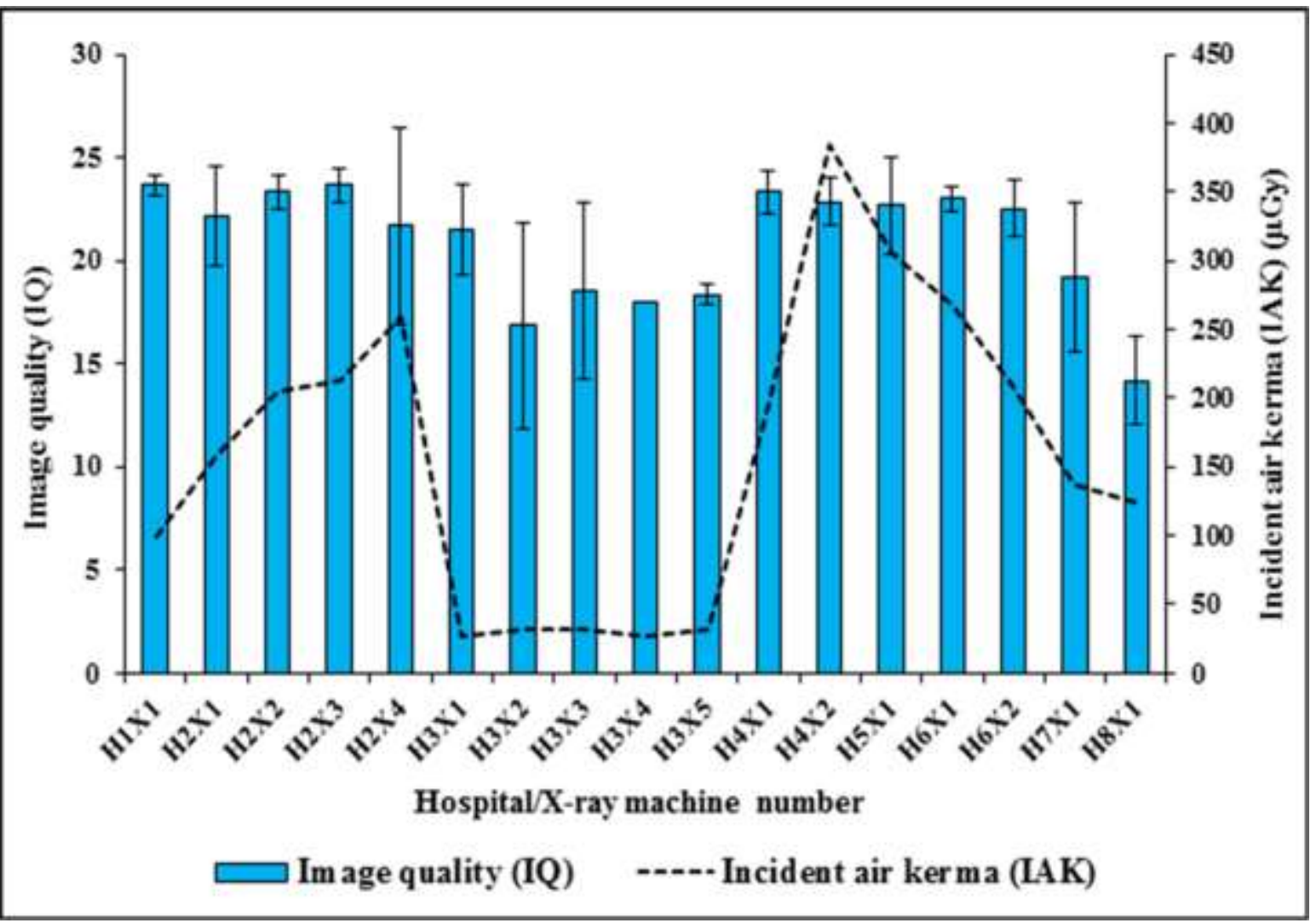

Fig. 3. A comparison of IQ against IAK for the larger size phantom images. The error bars in this chart represent the SD in IQ obtained from the visual evaluations. The dashed line represents the average IAK value that resulted from measuring three repeated radiation exposures. $\mathrm{H}=$ hospital; $\mathrm{X}=\mathrm{x}$-ray machine. 


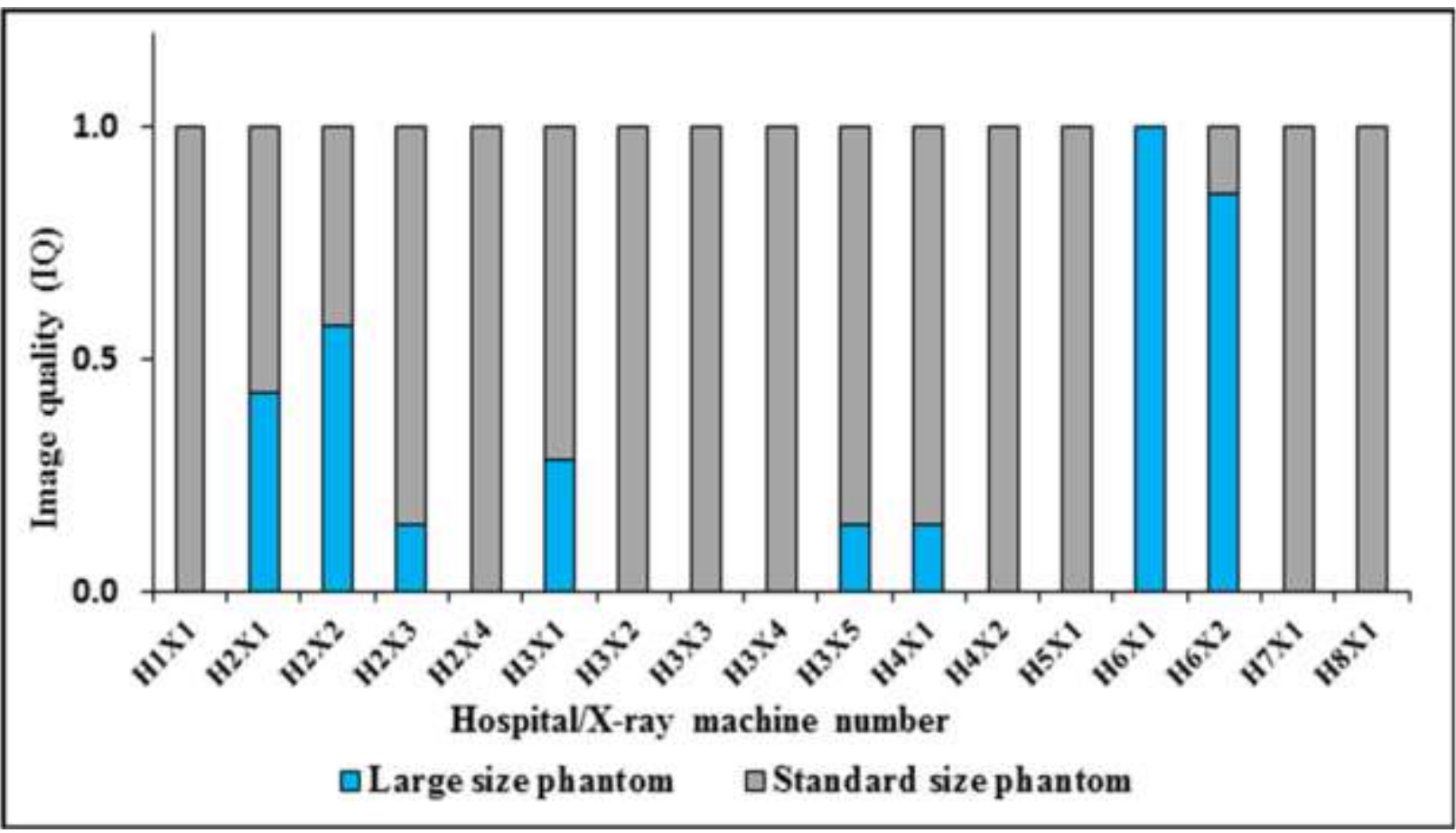

Fig. 4. 2AFC IQ evaluation scores on images generated using the standard and larger size Lungman phantoms. $\mathrm{H}=$ hospital; $\mathrm{X}=\mathrm{x}$-ray machine. 


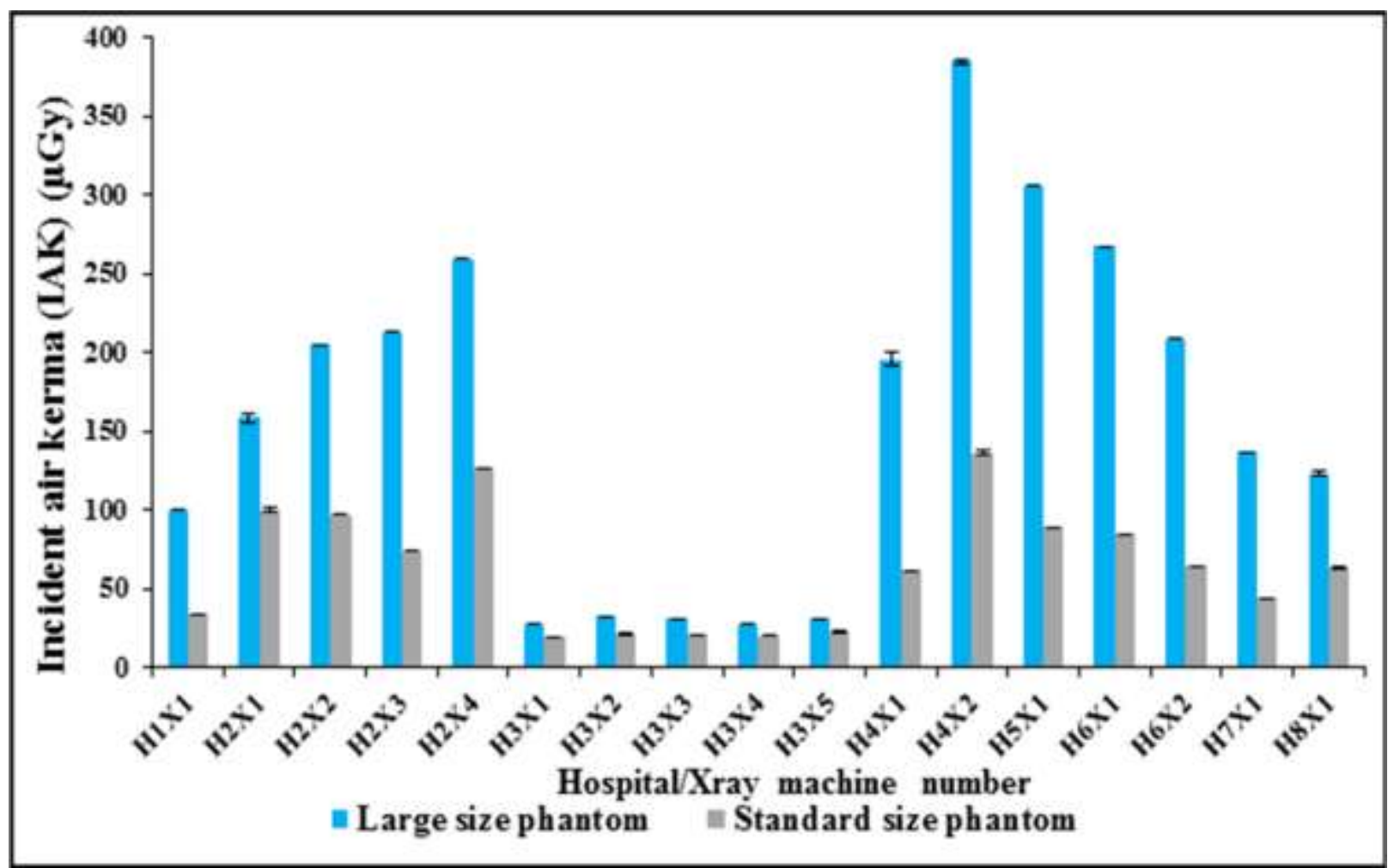

Fig. 5. The IAK values from images generated from the standard and larger size Lungman phantoms. The error bars in this chart represent the SD in IAK obtained from measuring three repeated radiation exposures. $\mathrm{H}=$ hospital; $\mathrm{X}=\mathrm{x}$-ray machine. 


\section{Discussion}

Our findings show that the inclusion of the chest plates has an influence on IQ and IAK. For IQ (Fig. 4) the larger sized phantom has a lower IQ compared with that of the standard size phantom for the majority of the $\mathrm{x}$-ray machines (thirteen $\mathrm{x}$-ray machines); this achieved statistical significance $(\mathrm{P}=0.001)$. This IQ reduction is expected due to the additional soft tissue thickness which leads to an increase in the attenuation of the incident $\mathrm{x}$-ray beam along with the production of additional scatter [4].

Another possible reason for the lower IQ obtained from the larger size phantom might be attributed to the majority of hospitals using relatively similar protocols (except mAs values) for both the standard and larger size phantom/patients; this is surprising because no adaptation was being made to their routine imaging protocols as can be seen from Table 1. Using a standard size PA chest protocol for a larger size patient could be lead to insufficient exposure reaching the image detector and result a suboptimal IQ which might have a negative influence on pathology identification. This is especially true when the examination is undertaken using manual exposure control compared with AEC [25].

Regarding the nine x-ray machines that utilised AEC (Table 1), all these x-ray machines used exactly the same protocols and acquisition parameters (except mAs values) for both the standard and larger size phantoms. The percentage increase in mAs, when the larger size phantom was imaged compared to the standard size, varied from $100.0 \%$ to $212.5 \%$ (Table 1). The $\mathrm{kVp}$ values were the same for both standard and larger size phantoms except only one x-ray machine (H2X4) that changed the $\mathrm{kVp}$; here $\mathrm{kVp}$ was increased from 125.00 for standard size phantom to 130.0 for the larger size phantom. With respect to the eight $\mathrm{x}$-ray machines that used manual exposure control (Table 1), the percentage increase in mAs, when moving from imaging the standard size phantom to the larger size phantom, ranged from $25.0 \%$ to $60.0 \%$, while the $\mathrm{kVp}$ values ranged from $0.0 \%$ to $9.0 \%$ (Table 1). X-ray machine H5X1 used AEC and anti-scatter grid with the larger size phantom instead of manual exposure control without anti-scatter grid for imaging the standard size phantom; this resulted in $12.9 \%$ and $117.1 \%$ increase in $\mathrm{kVp}$ and $\mathrm{mAs}$ for the larger size phantom, respectively (Table 1). From Fig. 4, two X-ray machines (H6X1 and H6X2) had better IQ for the images obtained from the phantom with chest plates compared with that of the standard size phantom; however, these images were associated with higher IAK (216\% and $224 \%$ percentage increase, H6X1 and H6X2, respectively). This could be due to the high mAs values 
recorded from the AEC with the larger size phantom compared with that of the standard size phantom $-192.8 \%$ and $200.0 \%$ increase for X-ray machines H6X1 and H6X2, respectively (Table 1). The high mAs values associated with the larger size phantom led to improved IQ. It has been demonstrated from the literature that there is a direct relationship between mAs and the IQ [2628]. Two X-ray machines $\mathrm{H} 2 \mathrm{X} 1$ and $\mathrm{H} 2 \mathrm{X} 2$ (Fig. 4) were found to have a similar IQ for the standard and larger size phantom images. For H2X1, this system used manual exposure control during imaging of both the standard and larger size phantom; the percentage increase for $\mathrm{kVp}$ and $\mathrm{mAs}$ for standard size to the larger size phantom were $9.1 \%$ and $42.8 \%$, respectively (Table 1).

The combined radiation dose values of $\mathrm{H} 2 \mathrm{X} 1$ for the standard size and larger size phantoms were $99 \mu \mathrm{Gy}$ and $158 \mu \mathrm{Gy}$ with a $59 \%$ percentage increase. This might be related to the mAs value recorded by the AEC when imaging the larger size phantom; $2.4 \mathrm{mAs}$ compared with $1.2 \mathrm{mAs}$. This constitutes a $100.0 \%$ increase (Table 1).

From our results, utilising the AEC was not always helpful in obtaining satisfactory IQ when the larger size phantom was used.

More research is needed to investigate how different acquisition parameters can influence IQ when larger size patients are imaged. Several studies have sought to establish strategies for achieving dose optimisation in adult CXR [29-31]. All of these focused on average sized patients did not consider optimisation for overweight and obese patients. Only a limited number of phantom studies have been conducted to investigate the impact of acquisition parameters on IQ and radiation dose for the larger body size patients in CXR [9,32]; however, these studies investigated the influence of only some parameters and did not cover all the acquisition parameters.

Regarding radiation dose, our results (Fig. 5) demonstrate that the additional fat thickness increases the radiation dose by $151 \%$ compared with that of the standard phantom size across all of the $\mathrm{x}$ ray machines with a statistical significant difference $(\mathrm{P}=0.002)$. Comparable results regarding the influence of additional fat thickness on radiation dose were reported in study by Otto et al. [32] and study be Perez et al. [33]. In our study, the larger size phantom was observed to have both higher IAK and lower IQ compared with that of the standard size phantom. Interestingly, the higher IAK received by the larger size phantom did not considerably improve IQ in most of the x-ray machines compared with standard size phantom and this emphasise that the protocols used for larger size phantom need further optimisation. 
Fig. 2 and 3 illustrate a wide variation in IQ and radiation dose for both standard size and larger size phantoms, between and within hospitals. This might be attributed to the high variability of the technical characteristics of image detector types (CR, DDR and IDR systems) and X-ray generators used between and within hospitals (Table 1). Several studies concluded that the DR systems can produce images with higher image quality compared with that obtained from CR systems, under similar dose levels [33,34] and this can be attributed to DR having a higher detective quantum efficiency (DQE) when compared with CR [35,36]. It is accepted that CR systems need more radiation, when compared with DR, to obtain a similar IQ and literature has reported that DR systems are significantly better than CR in dose minimisation, with possible dose decreases of up to $75 \%$ reported in comparison with the CR [34,37,38].

Furthermore, there was a considerable variation in technique and acquisition parameters such as $\mathrm{mAs}, \mathrm{kVp}$, additional filtration and anti-scatter radiation grid utilised for the same examination, between and within hospitals (Table 1); all of these have a direct influence on IQ and radiation dose $[27,34,35]$. In addition, the variability in IQ and radiation dose among $\mathrm{x}$-ray machines could also be attributed to the x-ray machine age since the ageing of the equipment can influence radiation output and consequently IQ.

The clinical effects of the observed variations in IQ for both standard and larger phantom sizes between and within hospitals and also the IQ variations resulted from the influence of phantom size in this study, should be taken into account in future work to help increasing the pathology detectability performance. These variations in IQ could impact on the detection of subtle pathology in clinical practice. Further study is needed to determine how these variations in IQ can affect pathology detection. If the detected differences in IQ described in this study have no negative influence on the diagnostic accuracy of pathology, then more reduction of the radiation dose can be reached.

Our study has some limitations. IQ evaluation was based on the visibility of the anatomical structures only and not did include the identification of pathology. Future work should include pathology identification /diagnostic performance with utilising a flexible continuous scale during image scoring instead of Liker scale (ordinal scores). Our study was carried out using a limited number of x-ray machines and it should be extended to include a larger sample of hospitals and $\mathrm{x}$ ray machines. It must be acknowledged that utilising a phantom to obtain the images raises some 
issues since they are limited by lack of movement and anatomical variation, which would be apparant in a patient population. Furthermore, the chest plates added to the Lungman phantom only simulates subcutaneous fat; visceral fat therefeore needs thought in future work.

\section{Conclusion}

The findings of our study demonstrate that CXR for the larger size phantom has lower IQ compared with that of the standard size phantom, for the majority of the x-ray units between and within the participating hospitals. The IAK was found to be higher for the larger size phantom and double that of the standard size phantom, for all the $\mathrm{x}$-ray units between and within the participating hospitals. Our data suggests that when using routine clinical CXR protocols for larger patients, IQ may be compromised (possibly resulting in reduced pathology detection) and radiation dose will be increased. Routine protocols may therefore require optimisation for this subcategory of patients. With the growing obesity and overweight rates, it is particularly important to establish imaging protocols designed for this category of patients.

\section{Acknowledgements}

The author is supported by the Higher Committee for Education Development in Iraq (HCEDIraq) and he gratefully acknowledges this supporting. 


\section{References}

[1] Eurostat Statistics Explained. Overweight and obesity - BMI statistics. Http://EcEuropaEu/ 2017. http://ec.europa.eu/eurostat/statisticsexplained/index.php/Overweight_and_obesity_-_BMI_statistics (accessed June 18, 2018).

[2] World Health Organization. Obesity : preventing and managing the global epidemic : report of a WHO consultation. World Health Organization; 2000.

[3] Hofmann B. Obesity as a Socially Defined Disease: Philosophical Considerations and Implications for Policy and Care. Heal Care Anal 2016;24:86-100. doi:10.1007/s10728015-0291-1.

[4] Carucci LR. Imaging obese patients: problems and solutions. Abdom Imaging 2013;38:630-46. doi:10.1007/s00261-012-9959-2.

[5] Yanch JC, Behrman RH, Hendricks MJ, McCall JH. Increased Radiation Dose to Overweight and Obese Patients from Radiographic Examinations. Radiology 2009;252:128-39. doi:10.1148/radiol.2521080141.

[6] Uppot RN, Sahani D V., Hahn PF, Gervais D, Mueller PR. Impact of obesity on medical imaging and image-guided intervention. Am J Roentgenol 2007;188:433-40. doi:10.2214/AJR.06.0409.

[7] Lazar MA, Plocher EK, Egol KA. Obesity and its relationship with pelvic and lowerextremity orthopedic trauma. Am J Orthop (Belle Mead NJ) 2010;39:175-82.

[8] Commission of the European Communities (CEC). European Guidelines on Quality Criteria for Diagnostic Radiographic Images:(EUR 16260 EN). Luxembourg: CEC: 1996.

[9] Moore CS, Avery G, Balcam S, Needler L, Swift A, Beavis AW, et al. Use of a digitally reconstructed radiograph-based computer simulation for the optimisation of chest radiographic techniques for computed radiography imaging systems. $\mathrm{Br} \mathrm{J}$ Radiol 2012;85:e630-9. doi:10.1259/bjr/47377285.

[10] Almén A, Lööf M, Mattsson S. Examination Technique, Image Quality, and Patient Dose in Paediatric Radiology. Acta Radiol 1996;37:337-42. doi:10.3109/02841859609177662.

[11] Geleijns J, Schultze Kool LJ, Zoetelief J, Zweers D, Broerse JJ. Image Quality and Dosimetric Aspects of Chest X Ray Examinations: Measurements with Various Types of Phantoms. Radiat Prot Dosimetry 1993;49:83-8. doi:10.1093/oxfordjournals.rpd.a081907.

[12] Veldkamp WJH, Kroft LJM, Boot M V., Mertens BJA, Geleijns J. Contrast-detail evaluation and dose assessment of eight digital chest radiography systems in clinical practice. Eur Radiol 2006;16:333-41. doi:10.1007/s00330-005-2887-6.

[13] Månsson LG, Båth M, Mattsson S. Priorities in optimisation of medical X-ray imaging - a contribution to the debate. Radiat Prot Dosimetry 2005;114:298-302. doi:10.1093/rpd/nch578.

[14] Institute of Physics and Engineering in Medicine. IPEM report 91: recommended standards for the routine performance testing of diagnostic x-ray imaging systems. York: IPEM; 2005. 
[15] Dewerd L.A KM. The Phantoms of Medical and Health Physics. New York.: Springer; 2014.

[16] Murata K, Catalog. Multipurpose Chest Phantom N1 "LUNGMAN." KyotokagakuCom 2018. https://www.kyotokagaku.com/products/detail03/ph-1.html.

[17] The Royal College of Radiologists. Picture archiving and communication systems (PACS) and guidelines on diagnostic display devices. 2012.

[18] Ma WK, Hogg P, Tootell A, Manning D, Thomas N, Kane T, et al. Anthropomorphic chest phantom imaging - The potential for dose creep in computed radiography. Radiography 2013;19:207-11. doi:10.1016/j.radi.2013.04.002.

[19] Allen E, Hogg P, Ma WK, Szczepura K. Fact or fiction: An analysis of the $10 \mathrm{kVp}$ 'rule' in computed radiography. Radiography 2013;19:223-7. doi:10.1016/j.radi.2013.05.003.

[20] M nsson LG. Methods for the Evaluation of Image Quality: A Review. Radiat Prot Dosimetry 2000;90:89-99. doi:10.1093/oxfordjournals.rpd.a033149.

[21] Cohen J. Statistical power analysis for the behavioral sciences.Hilsdale." NJ: Lawrence Earlbaum Associates 2. 1988.

[22] Filed A. Discovering statistics using SPSS statistics. Third edit. Londan: Sage publications Ltd; 2009.

[23] Koo TK, Li MY. A Guideline of Selecting and Reporting Intraclass Correlation Coefficients for Reliability Research. J Chiropr Med 2016;15:155-63. doi:10.1016/j.jcm.2016.02.012.

[24] Portney L, Watkins M. Foundations of clinical research: applications to practice.New Jersey: Prentice Hall. 2000.

[25] Buckley O, Ward E, Ryan A, Colin W, Snow A, Torreggiani WC. European obesity and the radiology department. What can we do to help? Eur Radiol 2009;19:298-309. doi:10.1007/s00330-008-1154-z.

[26] Saito K, Hiramoto S, Gomi T, Muramoto N, Seki M, Tsukimura K, et al. Evaluation of chest and abdominal exposure dose appropriate for a digital image reader system incorporating a columnar-crystal structured phosphor plate and a contrast-detail phantom. Radiol Phys Technol 2008;1:238-43. doi:10.1007/s12194-008-0035-0.

[27] Precht H, Tingberg A, Waaler D, Outzen CB. New Developed DR Detector Performs Radiographs of Hand, Pelvic and Premature Chest Anatomies at a Lower Radiation Dose and/or a Higher Image Quality. J Digit Imaging 2014;27:68-76. doi:10.1007/s10278-0139635-2.

[28] De Hauwere A, Bacher K, Smeets P, Verstraete K, Thierens H. Analysis of image quality in digital chest imaging. Radiat Prot Dosimetry 2005;117:174-7. doi:10.1093/rpd/nci748.

[29] Hamer OW, Sirlin CB, Strotzer M, Borisch I, Zorger N, Feuerbach S, et al. Chest radiography with a flat-panel detector: image quality with dose reduction after copper filtration. Radiology 2005;237:691-700. doi:10.1148/radiol.2372041738.

[30] Dobbins III James T., Samei E, Chotas HG, Warp RJ, Baydush AH, Floyd CE, et al. Chest 
radiography: optimization of X-ray spectrum for cesium iodide-amorphous silicon flatpanel detector. Radiology 2003;226:221-30. doi:10.1148/radiol.2261012023.

[31] Grewal RK, Young N, Colins L, Karunnaratne N, Sabharwal N. Digital chest radiography image quality assessment with dose reduction. Australas Phys Eng Sci Med 2012;35:7180. doi:10.1007/s13246-012-0125-5.

[32] Otto D, Ludwig K, Fessel A, Bernhardt TM, Kästner A, Reißberg S, et al. Digital selenium radiography: detection of subtle pulmonary lesions on images acquired with and without an additional antiscatter grid. Eur J Radiol 2000;36:108-14. doi:10.1016/S0720048X(00)00265-5.

[33] Rodríguez Pérez S, Marshall NW, Struelens L, Bosmans H. Characterization and validation of the thorax phantom Lungman for dose assessment in chest radiography optimization studies. J. Med. Imaging, vol. 5, 2018, p. 1. doi:10.1117/1.JMI.5.1.013504.

[34] Ween B, Olstad M, Jakobsen JÅ, Olsen DR. Pediatric digital chest radiography, comparison of grid versus non-grid techniques. Eur J Radiogr 2009;1:201-6. doi:10.1016/j.ejradi.2010.09.002.

[35] Ekpo EU, Hoban AC, McEntee MF. Optimisation of direct digital chest radiography using $\mathrm{Cu}$ filtration. Radiography 2014;20:346-50. doi:10.1016/j.radi.2014.07.001. 